\title{
SOBRE LOS PROBLEMAS GEOMÉTRICOS REALISTAS DE LA PRATICA MERCANTÍUOL (1521) DE JOAN VENTALLOL
}

\author{
Vicente Meavilla Seguí \\ Universidad de Zaragoza - España \\ Antonio M. Oller Marcén \\ Centro Universitario de la Defensa, Academia General Militar - España
}

(aceito para publicação em março de 2016)

\begin{abstract}
Resumo
La Pratica mercantíuol, escrita en catalán por el mallorquín Joan Ventallol, se imprimió en 1521. Estructurada en seis tratados, la Pratica es un ejemplo de las muchas aritméticas sin contenido algebraico que proliferaron a lo largo y ancho del siglo XVI. El sexto tratado se consagra a la Geometría elemental e incluye una colección de problemas de enunciado verbal con ilustraciones que tienen un cierto nivel artístico. En este artículo, comparamos dichos problemas con los que se proponen en otros impresos anteriores de carácter aritmético-geométrico. Con ello pretendemos descubrir las posibles fuentes en las que se pudo inspirar el autor balear.
\end{abstract}

Palabras clave: Problemas geométricos; Siglo XVI; Joan Ventallol; Pratica mercantíuol; fuentes impresas.

\section{[ON REALIST GEOMETRICAL PROBLEMS OF JOAN VENTALLOL'S PRATICA MERCANTÍNUOL (1521)]}

\begin{abstract}
The book Pratica mercantíuol written in Catalan by Joan Ventallol from Mallorca, was printed in 1521. Composed by six treaties, the Pratica is an example of the many arithmetic books without algebraic content that proliferated during the XVI century. The sixth treaty is about elementary geometry and includes a collection of story problems with illustrations that have some artistic level.
\end{abstract}


In this article we compare these problems with those in other earlier printings of arithmeticgeometric character. With this we strive to find possible sources in which the author from the Baleares could find inspiration.

Keywords: Geometric problems; XVI century; Joan Ventallol; Pratica mercantíuol; printed sources.

\section{Introducción}

Durante el siglo XVI, los nuevos descubrimientos geográficos, la proliferación de ferias y mercados, el aumento de instituciones dedicadas a comerciar con el dinero, etc., propiciaron la aparición de un buen número de manuales de matemáticas, las famosas Aritméticas, dirigidas a un público no matemático ni universitario y escritas en lengua vernácula.

En la mayoría de ellas, además de los capítulos dedicados al sistema de numeración decimal, a los algoritmos de las operaciones elementales con enteros y fracciones (adición, sustracción, multiplicación, división, extracción de la raíz cuadrada y de la raíz cúbica), y a la regla de tres, se presentaba, sin justificación alguna, un catálogo de trucos para que los lectores pudiesen acceder a la solución de toda una serie de problemas de carácter aritmético: reglas de compañía, aligación, repartos proporcionales, barata, regla de falsa posición simple, regla de dos falsas posiciones, etc. En otras, además del Arte Menor, se ofrecían los rudimentos del álgebra simbólica (Arte Mayor). Finalmente, algunas incluían también nociones básicas de Geometría.

Una obra de este tipo es la Pratica Mercatíuol, escrita en catalán en 1521 y que se incluye en el grupo de aritméticas comerciales que gozaron de una gran popularidad entre los tratantes y mercaderes del siglo XVI. Sin embargo, la Pratica no es solamente una aritmética comercial sin contenido algebraico y con nociones básicas de Geometría, sino un tratado que está a medio camino entre los puramente prácticos y los exclusivamente especulativos.

El autor de la Pratica mercantívol fue el mallorquín Joan Ventallol. Aunque Docampo (2006) presenta argumentos respecto a la autoría por parte de Ventallol de un manuscrito anónimo de contenido algebraico hallado en el Monasterio de San Cugat, datado en la primera parte del siglo XVI, lo cierto es que la Pratica es la única obra que podemos atribuir con total certeza a este autor.

De la biografía de Joan Ventallol ${ }^{1}$ sólo sabemos que era mallorquín, tal como se detalla en la portada y el colofón de su Pratica mercantíuol ${ }^{2}$.

${ }^{1}$ Según Pedro Ventayol Suau (1974), farmacéutico e historiador mallorquín, el apellido Ventayol es de origen catalán y todavía subsiste en Cataluña con leves modificaciones como Ventalló, Ventallol y Ventallons. De Cataluña pasó a Alcudia, sobre el año 1397. Según Francisco Piferrer (1858), el apellido Ventayol es originario de Monells (Gerona) de donde pasó a Alcudia y de allí a Ciudadela (Menorca). 


\section{Ifasticamercantiuol compofta eozdenadaper en zoanvatallo! delaciutat demalloantus}

Figura 1. Pratica mercantíuol. Detalle de la portada.

Aparte de esta información de primera mano, no se conocen otros aspectos de su vida o de su obra. De hecho, las notas biobibliográficas que hemos localizado se limitan a presentar de forma más o menos condensada esta información:

VÉNTAYOL (Juan), mallorquín instruidísimo en las matemáticas. Hablan de él el cronista D. Vicente Mut, Vargas de Ponce, Serra y el P. Pascual. Escribio: Practica mercantivol composte e ordenada per Johan Ventayol de la ciutat de Mallorques, un tomo en $4^{\circ}$ que concluye asi: En nom de N. S. Deu acabe la arithmetica y gesmetrie composte e ordenade per Johan Ventayol, natural de la ciutat de Mallorques, y á ses despeses estampada en la ciutat de Lyo, per mestre Johan de la Plaça, e acabada á 23 de abril de 1521. (BOVER DE ROSSELLÓ, 1838, p. 457.)

\section{Trtennoinoe noftro ESenyor oetuy oela'gloziafa'verge mazla yoe madona fancta Tozacedis patrona noftra. Acabala aritbmericae geometrta compofte ordonade per en Koun Eantalol natural De la Eutata oe EOSallozques pares oefperes ftampada en ta Lini tat oe Z poper meftre 3oan oc la place eacabadaa .23, oe Alpra anp.25210}

Figura 2. Pratica mercantíuol. Colofón. Adviértase el error en el año de impresión.

"Juan Ventayol fue sabio y profundo matemático, y sus obras se imprimieron en Leon en 1521”. (PIFERRER, 1858, p. 31).

"JUAN VENTALLOL. Mallorquín instruidísimo en las matemáticas y muy elogiado de los cronistas Mut, Serra, Vargas Ponce y del P. Pascual.

${ }^{2}$ Aunque en la Pratica mercantíuol encontramos el apellido de su autor escrito de dos formas distintas (Vantallol y Vantalol), a lo largo de este trabajo utilizaremos la «variante" Ventallol con la que se le cita en la literatura especializada. 
Escribió Practicas mercantivol composta e ordenada per en Joan Ventallol de la ciutat de Mallorques. Este título está bajo de un escudo de las armas que contienen las águilas imperiales con los blasones de Castilla y León en el ala derecha, y los de Mallorca en la izquierda. Un vol., en $4^{o}$ de 133 hojas, foliación romana y varios grabados.

Esta obra fue editada a sus expensas en la ciudad de Lión por el impresor Juan de la Place y acabada el 23 de abril de 1521, y de ella reconoce haberse servido el Dr. Antich Roca de Gerona, para escribir su Aritmética que publicó en Barcelona, imprenta de Claudio Bornat el año 1564.

Anteriormente ya la había traducido al castellano el Dr. Juan Bautista Tolrá, que dióla a la estampa en Lión el año 1524. De esta edición de Tolrá hay una segunda de Zaragoza, imprenta de Gabriel Roberto, con el título La Aritmética de Juan Ventallol traducido de lengua catalana en castellano, añadido un tratado de Algebra, dedicada al Conde se Savellá ${ }^{3}$. De este libro tan importante conserva un ejemplar Don Luis Alemany, en Palma”. (VENTANYOL SUAU, 1974, p. 323)

La Pratica Mercatíuol se desarrolla a lo largo de ciento treinta y dos folios ${ }^{4}$ y se estructura en seis tratados cuyos contenidos se detallan a continuación:

\begin{tabular}{ll}
\hline Primer tratado & $\begin{array}{l}\text { Definición de unidad y número. Números pares e impares. Número } \\
\text { pariter par, pariter impar, impariter par, defectuoso, abundante, } \\
\text { perfecto, primo, compuesto. Números primos entre sí. }\end{array}$ \\
\hline Segundo tratado & Numeración. Valor de las monedas y los pesos de algunas tierras. \\
& Adición, sustracción, multiplicación y división de números naturales \\
& y quebrados. Reglas breves para reducir monedas de una especie en \\
& otra.
\end{tabular}

Tercer tratado Regla de tres simple. Reducción de monedas. Regla de tres

\footnotetext{
${ }^{3}$ No conocemos la edición de 1524 ni la de Zaragoza.

${ }^{4}$ Debido a errores en la foliación, el último folio del original es el 133v. En la fe de erratas (Taula de correctio dela present obra), Ventallol, con cierta ironía, puntualiza: «Lo qui volra corregir la present hobre es manaster primer coregesca lo nombre deles fules". (El que quiera corregir la presente obra es preciso que primero corrija la numeración de las hojas). A pesar de los errores, respetaremos la foliación original cuando tengamos necesidad de localizar y ubicar citas, textos, etc.
} 
compuesta. Regla de censales. Regla de taras. Regla de tres con

tiempo. Regla de compañía. Compañías con tiempo. Baratas. Baratas

con tiempo. Cambios. Méritos. Reglas de viajes. Aligaciones.

Progresiones. Regla de una falsa posición. Regla de dos falsas

posiciones.

\begin{tabular}{|c|c|}
\hline Cuarto tratado & $\begin{array}{l}\text { Extracción de la raíz cuadrada y la raíz cúbica. Reglas de cuadratura. } \\
\text { Testamentos. Reglas extraordinarias. }\end{array}$ \\
\hline Quinto tratado & $\begin{array}{l}\text { Proporciones (multiplex, superparticular, superpartiente, multiplex } \\
\text { superparticular, multiplex superpartiente). De las siete maravillas de } \\
\text { las proporciones. Sumar, restar, multiplicar y dividir en proporción. } \\
\text { Reglas proporcionales. Regla de mediación entre el más y el menos. } \\
\text { Juegos de adivinación por la ciencia de los números. }\end{array}$ \\
\hline Sexto tratado & $\begin{array}{l}\text { Definición de los números figurados. Medición de tierras según la } \\
\text { práctica de Barcelona. Práctica de la geometría según lo que han } \\
\text { escrito otros prácticos. Determinación del centro de un arco de } \\
\text { circunferencia. }\end{array}$ \\
\hline
\end{tabular}

\section{Objetivos y método}

El gran número de obras de características similares a la anterior hace que, pese a las dificultades evidentes (BEAUJOUAN, 1988), sea de interés el estudio de las posibles influencias entre manuscritos relativamente contemporáneos (LAMASSÉ, 2015).

Usón (2004) señala que este tipo de estudios requieren de un análisis profundo de los textos considerados y, de hecho, son pocos los trabajos en esta línea. Labarthe (2004) realiza un detallado estudio comparado de las obras de Santcliment, Ortega y Ventallol. Massa (2012), por su parte, estudia la influencia de Marco Aurel y Pérez de Moya sobre Antich Roca. Finalmente, Céu (2013) analiza las fuentes renacentistas de las obras geométricas de Pérez de Moya. En este trabajo pretendemos abordar un estudio comparativo centrado en la parte geométrica de la Pratica Mercatíuol de Joan Ventallol (1521).

La parte geométrica de la obra se desarrolla, como hemos visto, en el sexto tratado de la Pratica mercantíuol que "tracta delart de geometria" y ocupa doce folios (122r-133r). Contiene una breve introducción dedicada a los números "secundum figuram" (números laterales, superficiales, sólidos, cuadrados, cubos, triangulares y circulares) y cincuenta y siete reglas en las que se presentan distintos procedimientos para la medida de tierras de diferentes formas y se proponen problemas geométricos clásicos de enunciado verbal (problema del bambú roto, torres y escaleras, etc.). La mayor parte de los problemas 
propuestos por el mallorquín en este tratado se presentan en un contexto puramente matemático; por ejemplo, calcular la altura de un trapecio isósceles de lados 6, 40, 40 y 60 (Regla 13, folio 125r.). Sin embargo, en las reglas 41 a 55 y en la regla 57 los problemas se presentan en un contexto realista (Díaz y Poblete, 2001), con un enunciado verbal y acompañados de ilustraciones artísticas. Los enunciados de dichos problemas se complementan con ilustraciones artísticas que no aparecen en aritméticas españolas anteriores 5 .

El estudio que abordamos se centra en estos 16 problemas verbales de contexto realista y persigue dos objetivos fundamentalmente:

1. Presentar estos problemas en detalle dado su carácter relativamente excepcional (menos de un tercio del totoal) dentro del conjunto del sexto tratado.

2. Comparar estos problemas con los presentes en otras obras anteriores de características similares a la de Ventallol.

\begin{tabular}{lcl}
\hline \multicolumn{1}{c}{ OBRA } & AÑO DE PUBLICACIÓN & \multicolumn{1}{c}{ AUTOR } \\
\hline $\begin{array}{l}\text { De arimethrica opusculum } \\
\begin{array}{l}\text { Summa de Arithmetica, Geometria, } \\
\text { Proportioni et Proportionalita }\end{array}\end{array}$ & 1491 & Filippo Calandri \\
$\begin{array}{l}\text { Conpusicion de la arte de la } \\
\text { arismetica y juntamente de } \\
\text { geometria }\end{array}$ & 1494 & Luca Pacioli \\
$\begin{array}{l}\text { Tratado da pratica Darismetica } \\
\text { Larismethique nouellement } \\
\text { composee }\end{array}$ & 1512 & Juan de Ortega \\
\hline
\end{tabular}

En la tabla anterior se recoge el listado de obras utilizadas para la comparación. Todas ellas son aritméticas que contienen una sección consagrada a la geometría, están escritas en lengua vernácula y son textos que no se dirigen a un público universitario. Además de este motivo, las razones que motivan la elección de estas fuentes son las siguientes:

- Como hemos mencionado, Calandri y Nicolas incluyen ilustraciones artísticas como acompañamiento en sus obras; una práctica poco habitual y que puede denotar una posible influencia sobre Ventallol.

${ }^{5}$ Que sepamos, el primer impreso matemático europeo con ilustraciones artísticas es $D e$ arimethrica opusculum (CALANDRI, 1491). En la Península Ibérica, la primera Aritmética con ilustraciones artísticas es el Tratado da pratica Darismetica (Nicolas, 1519). la Pratica mercantíuol es, pues, el primer manual de Matemáticas editado en España con ilustraciones de este tipo. 
- La influencia de la obra de Pacioli sobre la de Ventallol es sobradamente conocida (Docampo, 2006, p. 45).

- En el segundo y tercer tratado de la Pratica mercantíuol Joan Ventallol cita a Juan de Ortega al referirse a asuntos concernientes a operaciones con números complejos, operaciones con quebrados, reglas de siete cantidades y cambios. Así pues, parece claro que Ventallol conocía la obra de Ortega.

- En el quinto tratado de la Pratica, se presenta la "retgla dita de mediacio entre lo mes e lo manco", que Estienne de la Roche también incluye en su obra. Además, el quinto tratado de la Pratica mercantíuol concluye con una sección consagrada a la Matemática Recreativa (Seguetxen se los iochs que per sciencia de nombres sembla que endiuine(n) moltes coses) cuyas coincidencias con Larismethique ponen de manifiesto una influencia de esta obra sobre el libro de Ventallol.

\section{Resultados del estudio}

Para cada uno de los 16 problemas analizados (que el autor denomina "reglas"), además de su enunciado original con su adaptación al castellano, presentaremos los problemas similares de las obras analizadas que se hayan encontrado y algunos comentarios.

\subsection{La Regla 41 (fol. 129v)}

El enunciado original del problema, que implica el cálculo del volumen de un tonel (bota) conociendo su altura y los diámetros superior, medio e inferior del tonel, es como sigue:

"Es vn bota q(ue) te d(e) alt p(er) un fo(n)s 5 palms ep(er) laltra fo(n)s te $7 \mathrm{pa}(\mathrm{m}) \mathrm{ls}$ y $\mathrm{p}(\mathrm{er})$ lo mig te 8 palms y de larc te $12 \mathrm{pa}(\mathrm{lm}) \mathrm{s}$ io(us) dema(n) qua(n)ts somades hi cabra araho de 4 palms quodrats $p($ er) somada”.

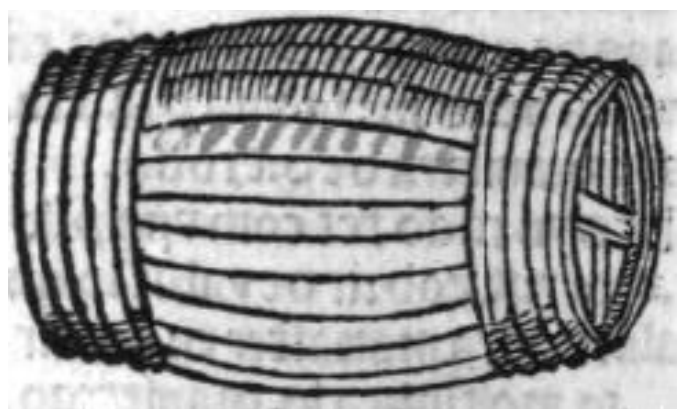

Figura 3. Ilustración correspondiente a la Regla 41. 
Hemos encontrado tres problemas similares en las obras de Calandri, Pacioli y Ortega. En concreto:

"Eglie una botte che il diamitro del fondo di dietro es 2 braccia $\tau$ nel mezo dal cuchiu(m)e e alta 2 braccia $\tau \frac{1}{2} \tau$ il diamitro del fo(n)do dina(n)zie 1 braccio $\tau \frac{1}{2}$ et dalluno fo(n)do allaltro e 3 braccia: uo sapere qua(n)to uino terra tene(n)do il braccio quadro 5 barrili". (CALANDRI, 1491, fol.92r).

"Eglie vna botte che ciascuno fondo e alto $1^{\circ}$ bracio $\frac{1}{3}$ e nel cocchiume e $1^{\circ}$ br(aci)o $\frac{1}{2}$. E da luno fondo alaltro e 2 bracia. Adimando qua(n)to vino tiene”. (PACIOLI, 1494, Tractatus Geometrie, fol. 52v).

"Si quisieres saber si vna cuba que tiene de alto por el vn te(n)pano 7 palmos: y por el otro tenpano 8 palmos y por el medio 10 palmos: y d(e) largo 6 palmos: quantas cantaras de vino cabra en ella: a razo(n) de 9 palmos quadrados cada cantara: faras ansi: ayunta la alteca delvn te(n)pano co(n) la alteca del otro y sumara(n) 15 de los quales 15 toma o quita la mitad y q(ue)dara(n) $7 \frac{1}{2}$ los quales $7 \frac{1}{2}$ aiu(n)ta con la altura de en medio como son 10 y serán $17 \frac{1}{2}$ de los quales $17 \frac{1}{2}$ quita tambien su mitad y quedaran 8 y tres quartos: los quales 8 y $\frac{3}{4}$ multiplica por si diciendo 8 y tres quartos multiplicados por 8 y tres quartos montaran 76 y пиеие sezabos delos quales 76 y nueue sezabos quita los 3 catorzenes q(ue) son 16 y nouenta y vno 224 abos y allaras que q(ue)dan 60 enteros y 5 treinta y dos abos de vn entero los quales 60 y 5 treinta y dos abos mutiplica por 6 y allaras que monta 360 y quinze sezenes de vn entero los quales todos son palmos quadrados (...)". (ORTEGA, 1512, fols. 107v-108r).

Ventallol calcula el valor aproximado de la capacidad de una cuba reemplazándola por un cilindro de la misma altura y cuyo diámetro es igual a:

$$
\frac{\frac{d_{s}+d_{i}}{2}+d_{m}}{2}
$$

siendo $d_{s}$ el diámetro superior, $d_{m}$ el diámetro medio y $d_{i}$ el diámetro inferior del tonel. De los tres autores que incluyen problemas similares, únicamente Ortega sigue un procedimiento de resolución similar. 


\subsection{La Regla 42 (fol. $129 v)$}

Este problema está relacionado con el anterior, pues involucra también en cierto modo el volumen de un tonel. En este caso se pretende estudiar la variación en el volumen que se produce al modificar cierto aspecto del tamaño del tonel:

"Es vna bota de 20 dogues totes de vna amplaria y largaria y caben hi 20 somades de vi ious daman Leuant ne dues dogues quant vi y cabra (...)".

Es una bota de 20 duelas de la misma anchura y longitud en la que caben 20 somadas de vino. Demando: si se quitan dos duelas, ¿cuánto vino cabrá?

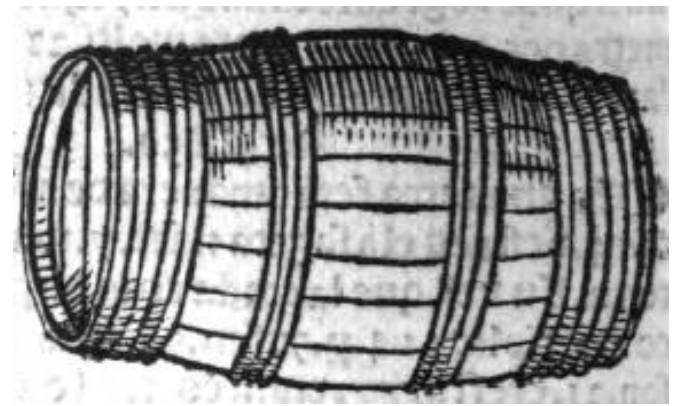

Figura 4. Ilustración correspondiente a la Regla 42.

En los manuales consultados sólo hemos descubierto un problema similar en la obra de Calandri:

"Una botte ha 50 doghe $\tau$ tiene 50 barili uo sapere unaltra botte che tenga 18 barili di qua(n)te doghe sara alla medesima ragio(n)e”. (CALANDRI, 1491, fol. 94v).

Se trata, efectivamente, de un problema relacionado con el volumen de toneles. Sin embargo es en cierto modo el problema inverso al propuesto por Ventallol ya que se solicita estudiar la variación que se produce en el tamaño del tonel al variar su volumen.

\subsection{La Regla 43 (fols. 129v-130r)}

Este problema trata del cálculo del volumen de una figura elipsoidal:

"Un puig radoal punt agut a fayso de vn paller e ha de la sumitat dedalt fins enterra mesurant a fayso darc 100 palms e lo seu peude baix gira entorn 80. ious deman quanta es aquesta terra". 

consultados.

No hemos encontrado ningún problema semejante en ninguno de los cinco textos

\subsection{La Regla 44 (fol. 130r)}

Los tres problemas que componen esta regla corresponden a las dos situaciones posibles que se pueden dar ante el teorema de Pitágoras; dados los catetos calcular la hipotenusa y dada la hipotenusa y un cateto calcular el otro. Además en los tres casos se trata del mismo triángulo de lados 6,8 y 10:

"Es vna torre alta 8 destres y sta valaiada ho en circuida de vn riu que te 6 destres de ample ious deman vna scala que bastas del peu del riu fins a la sima de la torra qua(n)t haura de larc (...)”.

“(...) Es vna torra alta 8 destres e te vn vall entorn ample que de la vora del vall fins ala sima de la torra amaster vna scala que tinga 10 destres de larc ious dema(n) qua(n)t te de ample (...)".

"Esit demanen q(ue) teni(n)t la scala 10 canes e lo val 6 qua(n)t deu tenir de alt la torra (...)”.

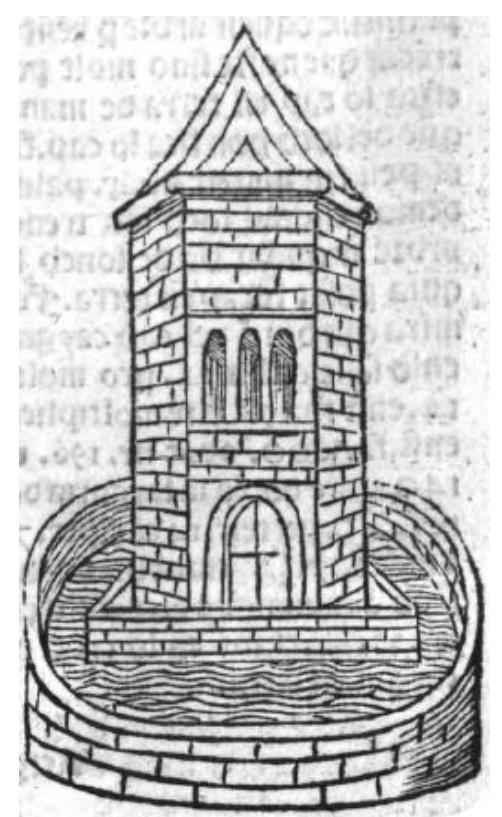

Figura 5. Ilustración correspondiente a la Regla 44. 
Hemos encontrado problemas similares a estos en todas las obras consultadas:

"Eglie una torre che e alta 40 braccia $\tau$ dappie uipassa uno fiume che e largho 30 braccia. uo sapere quanto sara lungha una fune che sia appicata alla riua del fiume $\tau$ alla cima della torre”. (CALANDRI, 1491, fol. 94r).

"Una torre e alta 40 braccia e dapie lipassa un fiume largo 30 braccia. Admandasi quanto sia dala cima dela torre infino alorlo del fiume”. (PACIOLI, 1494, Tractatus Geometrie, fol. 52v).

“(...) eglie vna torre alta 40 bra(ccia) e da pie li passa un fiume che non so quanto se largo: ma ben so che ponendo una fune dala cima dela torre infino alorlo del fiume e 50 bra(ccia). Adimandasi quanto e largo il fiume”. (PACIOLI, 1494, Tractatus Geometrie, fol. 53r).

“(...) eglie una torre che no(n) so qua(n)to e alta e da pie li passa un fiume largo 30 bra(ccia). E po(n)go una scala da lorlo del fiume infino ala cima dela torre laq(ua)le e 50 bra(ccia). Adima(n)do q(uan)to e alto la torre”. (PACIOLI, 1494, Tractatus Geometrie, fol. 53r).

"Un cauallero tiene vna torre la q(ua)l tiene 50 canas de alto: y quiere fazer vna escala $q($ ue) se aparte 40 canas desde el cimie(n)to dela torre: demando que qua(n)tas canas terna la escala dela pu(n)ta dela torre fasta el postrero pie de las 40 canas". (ORTEGA, 1512, fols. 202r-202v).

"Un hombre tiene vna casa fuerte la qual tiene 40 canas de alto este ho(m)bre tiene vna escala q(ue) tiene 60 canas de largo y qere la poner desde encima de la torre fasta la tierra demando que qua(n)tas canas haura desde el pie de la torre fasta la punta del pie de la escala". (ORTEGA, 1512, fol. 202v).

"He huu(n)a torre d(e) 40 braças estou arredrado d(e)la 30 p(re)gunto deitando huu(n)a lynha dos meus pees a po(n)ta da torre qua(n)to teria ha linha”. (NICOLAS, 1519, fols. 85r-85v).

"Ese te dixessem que era hu(n)a torre cuja altura nam he sabida porem sabemos que estamos arredrado della 30 braças $\tau$ a linha que vay donde nos estamos açima ha ponta da torre sabemos que he 50 braças demando quam alta he ha torre". (NICOLAS, 1519, fol. 85v).

"Il est vng mur qui a 30 piez de hault c(on)tre le quel on veult faire vne eschielle dont lu(n)g des boutz soit esloigne du mur 10 piez et laultre bout 
touche la summite du mur. Lon demande combien aura de long leschielle”. (de la Roche, 1520, fol. 226v).

"Et qui esloigneroit du meur le bout dembas de ladeschielle de 15 piez. A qua(n)t piez de halt du mur viendroit toucher le bout de dessus de ladeschielle". (de la Roche, 1520, fols. 226v-227r).

"Et qui co(n)tre le mur de 30 pyez de hault vouldroit appoyer vne eschielle dont q(ue) eust 35 pyes de long et que le bout de dessus de ladeschielle touchant la summite du mur. Lon dema(n)de a co(m)bien du mur fauldroit il esloigner le bout dembas de ladeschielle”. (de la Roche, 1520, fol. 227r).

Es interesante observar que únicamente Pacioli y de la Roche presentan los tres casos propuestos por Ventallol. Además, sólo en el texto de Pacioli el contexto es el mismo involucrando una torre rodeada por un río y el uso de una escalera.

\subsection{La regla 45 (fols. $130 r-130 v$ )}

Nuevamente se trata de una aplicación del Teorema de Pitágoras, en concreto del cálculo de la hipotenusa a partir de los dos catetos, aunque en este caso el valor de uno de los catetos no se proporciona explícitamente:

"Dues torres stan la vna dauant laltra la mes alta te 60 canes de alt la mes baixa te 40 canes de alt. E lo cami que sta en mig te 25 canes de ample ious deman quant ha de la vna sima de torra alaltra”.

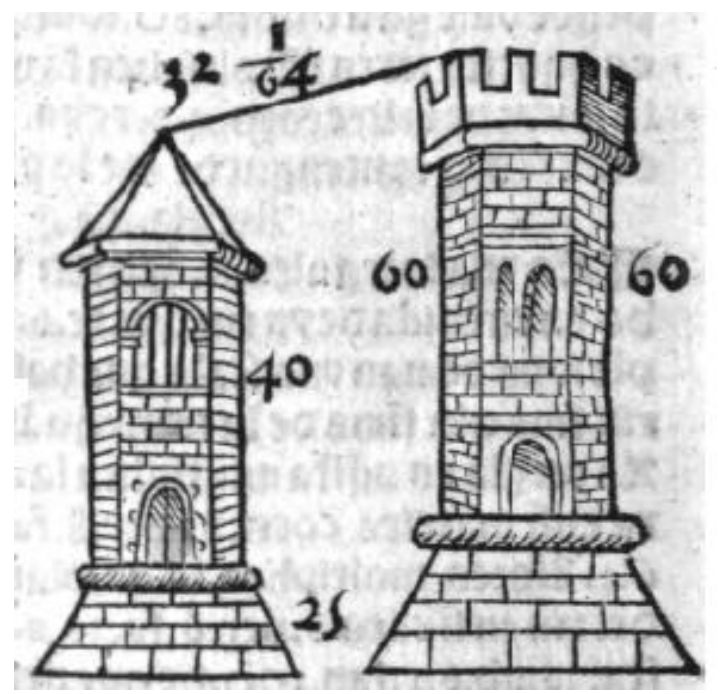

Figura 6. Ilustración correspondiente a la Regla 45. 
Hay problemas parecidos en las obras de Ortega y Nicolas ${ }^{6}$ :

"Un ho(m)bre tiene dos fortalezas en que la vna tiene 50 canas de alto y la segunda tiene 30 canas de alto: estas dos torres estan apartadas: la vna d(e)la otra 20 canas: y el dueño destas dos torres quiere facer vn passadizo d(e)la vna pu(n)ta dela torre fasta la otra pu(n)ta dela otra torre dema(n)do que qua(n)tas canas terna de largo el tal passadizo". (ORTEGA, 1512, fol. 202r).

"Sam duas torres nam yguaes daltura huu(n)a te(n) 20 braças dalto $\tau$ outra te(n) $15 \tau$ esta arredrada huu(n)a da outra 4 braças p(re)gu(n)to la(n)ça(n)do huu(na) lynha de ponta a po(n)ta por çima camanha sera". (NICOLAS, 1519, fol. 88r).

\subsection{La Regla 46 (fol 130v)}

En esta regla, Ventallol presenta una versión del "problema del bambú roto" que aparece en diferentes culturas (China, India, etc.) a lo largo de los tiempos y que está íntimamente relacionado con el teorema de Pitágoras. En realidad, el "problema del bambú roto" equivale a determinar la hipotenusa y un cateto de un triángulo rectángulo conociendo la suma de sus longitudes y la longitud del otro cateto:

"Un nauiliq(ue) sta tret e(n)terra elarbra te de terra fins ala sima 40 palms. Eaquest arbre p(er) vent ses tre(n)cat que noste sino molt poch efica lo cap en terra de manera que delloch hon fica lo cap fins al peu seu mateix a 14 palms io(us) deman enqual loch ses trencat dir arbre nequant ha de lonch lo tros quia posat lo cap en terra".

\footnotetext{
${ }^{6}$ «Sam duas torres nam yguaes daltura huu(n)a te(n) 20 braças dalto $\tau$ outra te(n) $15 \tau$ esta arredrada huu(n)a da outra 4 braças $\mathrm{p}(\mathrm{re}) \mathrm{gu}(\mathrm{n})$ to la(n)ça(n)do huu(na) lynha de ponta a po(n)ta por çima camanha sera". (fol. 88r).
} 


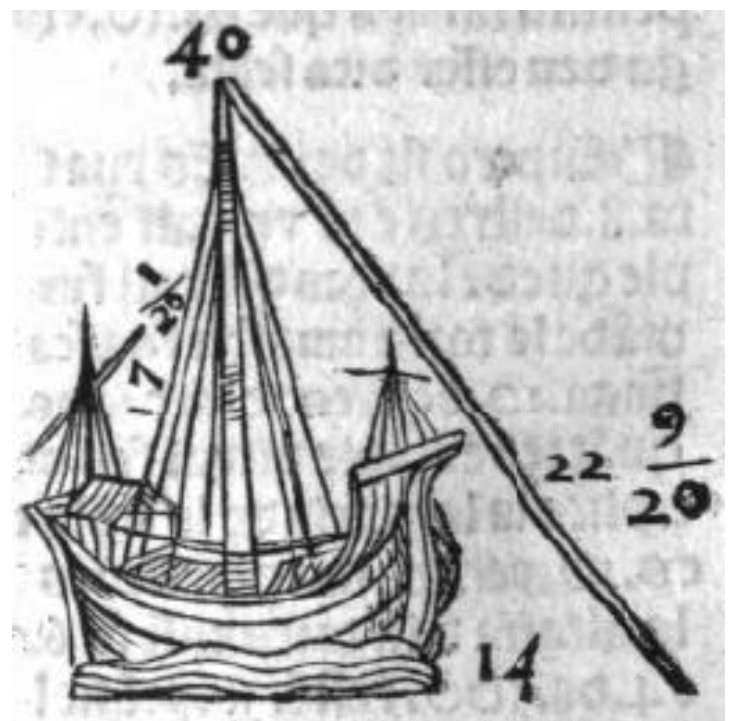

Figura 7. Ilustración correspondiente a la Regla 46.

Hemos encontrado enunciados del mismo problema, aunque en contextos diferentes, en las obras de Calandri, Pacioli y Gaspar Nicolas:

"Eglie un albero insu la riua dun fiume elquale e alto 50 braccia el fiume e largo 30 braccia $\tau$ per fortuna di ue(n)to siruppe intal luogo che la cima dellalbero toccaua lariua del fiume. Uo sapere quante braccia sene ruppe $\tau$ quanto nerimase ritto". (CALANDRI, 1491, fol. 94r).

"Egli vno albore alto 30 bracia talgliasi i(n)tal p(ar)te che lauetta sta iterra ap(re)sso alpedale a 10 bracia: e laltro capo e appiccato alalbore. Adimandasi $i$ che p(ar)te si ruppe lo detto albore”. (PACIOLI, 1494, Tractatus Geometrie, fol. 55r).

"He huu(n)a aruore de 50 braças $\tau$ esta ao pee de huu(n) rio que tem 30 braças de largura $\tau$ esta aruore quebrou por tal lugar que foy dar apo(n)ta alem na borda do rio: demando por onde quebrou”. (NICOLAS, 1519, fols. 86r-86v).

Aunque los contextos no coinciden, la resolución de Ventallol coincide con la propuesta por Filippo Calandri en su De arimethrica opusculum y se apoya en el teorema de Pitágoras a diferencia, por ejemplo, de lo que sucede en el texto de Pacioli se apoya en la Proposición 35 del libro III de los Elementos de Euclides. 
Este problema es idéntico al anterior salvo por el contexto en que se presenta, valen pues las mismas consideraciones que para el caso anterior:

"Un basto es alt de terra 70 palms e del cap del basto de terra fins a huna font quey ha ha 15 pams e dit basto se tre(n)ca en tal loch que lo cap del basto dona dins la font dema(n) qua(n)t es larch lo basto que resta".

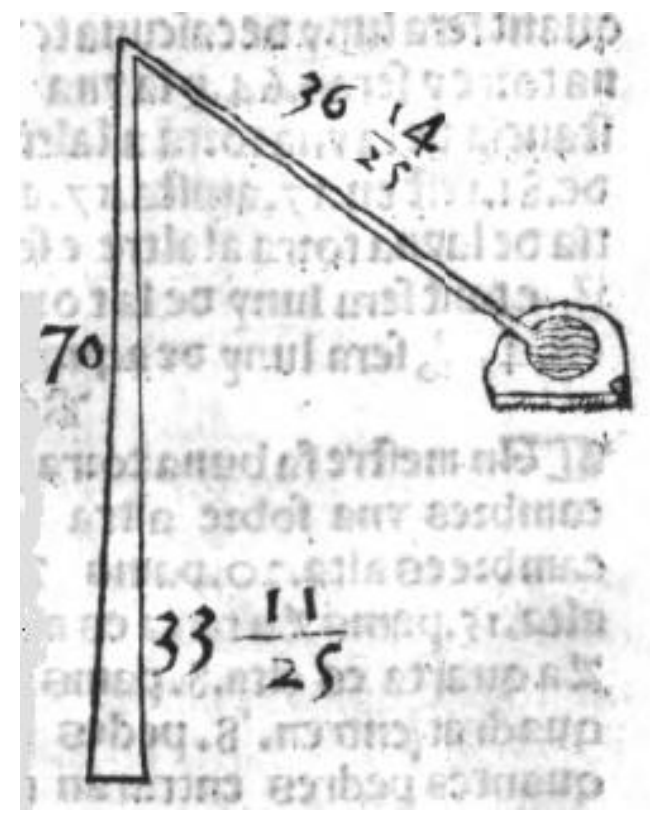

Figura 8. Ilustración correspondiente a la Regla 47.

\subsection{La Regla 48 (fol. 131r)}

Las dos cuestiones propuestas en esta regla son similares a dos de las tres que se plantean en la regla 44:

"Una torra en terra plana es alta 20 canes e luny de aquella 50 canes es plantat vn arbre ious deman qua(n)t ha de la sima dela torra fins al peu de larbre".

"Esit deyen que dela sima dela torra fins al peu de larbre a 40 canas quant haura del peu de larbre fins al peu de la torra per terra plana". 


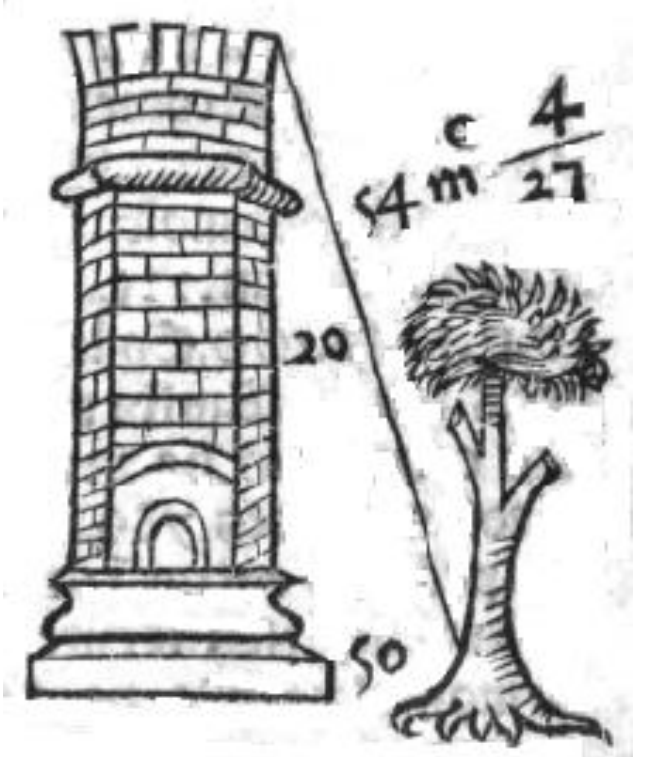

Figura 9. Ilustración correspondiente a la Regla 48.

En este caso (ver apartado 3.4.) tanto Pacioli, como Ortega y de la Roche contienen los dos casos enunciados por Ventallol.

3.9. La Regla 49 (fols. 131r-131v)

Este problema aparece ya en el Liber Abaci en un contexto muy similar:

"So(n) dues torres anun pla que de la vna alaltra ha 10 canes. Ela vna torre te 8 canes de alt elaltre ne te 9 y entra aquestes dues torres ha vna font ental loch q(ue) es tan luny dela vna sima de la vna torra com de lalatre io(us) deman quant sera luny de cascuna torre". 


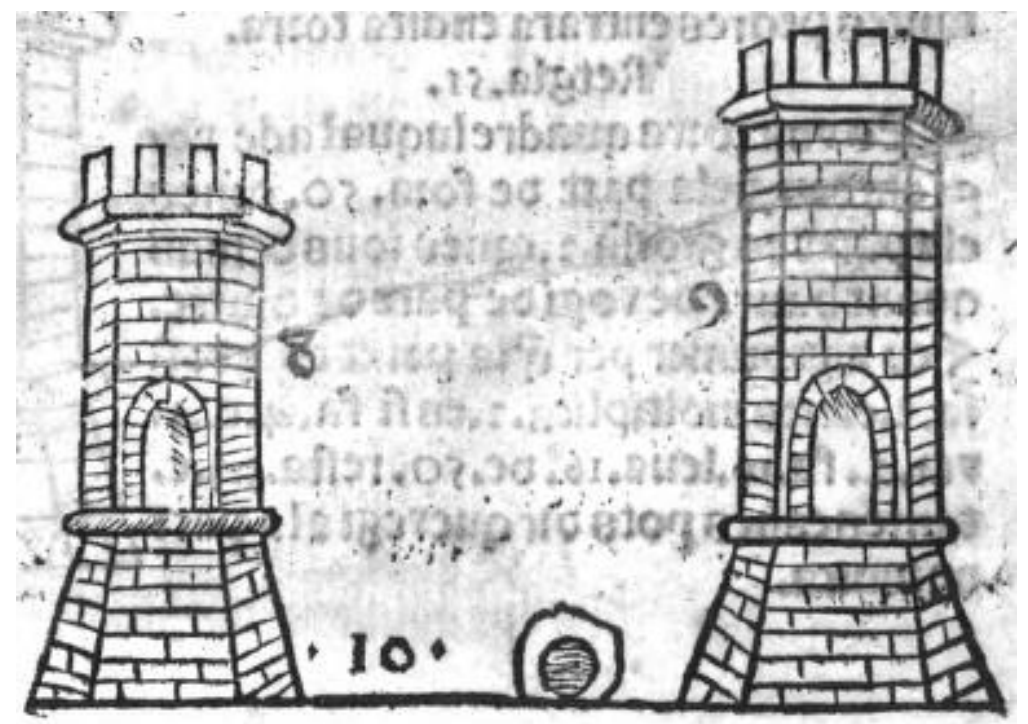

Figura 10. Ilustración correspondiente a la Regla 49.

Hemos encontrado enunciados del mismo tipo en las obras de Calandri, Pacioli y Nicolas:

"Esono dua torri in nun piano che luna e alta 80 braccia et laltra e alta 90 braccia: et dal luna torre allaltra e 100 braccia: et intra queste dua torri e una fonte dacqua in tal luogho che mouendosi due uccegli uno diciascuna et uolando dipari uolo guighono alla decta fonte aun tracto. Uo sapere quanto la fonte sara presso a ciascuna torre”. (CALANDRI, 1491, fol. $96 \mathrm{v})$.

“Eso(n)no 2 torri lu(n)a distante alaltra 150 e lu(n)a e alta cioe ab. 100 bra(cia) e cd. e 70 bracia e u(n) pia(no) bd. e 150 in su le quali torri cioe in ciascuna extremita e uno uccelo e auno tratto simouano e auno modo uolano e auno tratto gio(n)gano auna fonte situata in fral $b . e d$. adimandasi quella fonte quanto e apresso al b. e quanto e apresso al d". (PACIOLI, 1494, Tractatus Geometrie, fol. 59v).

"Sam duas torres huu(n)a de 90 braças $\tau$ outra de $80 \tau$ esta arredrada huu(n)a da outra 100 braças. E antre ambas has torres esta huu(n)a fonte em tal lugar que duas aues yguaes no voar ven beber aquella fo(n)te que cada hu(n)a das torres tem sua aue em cima $\tau$ partem ambas ha huu(n) tempo $\tau$ chegan ambas ha huu(n) tempo ha fonte. Demando 
quanto esta ha fonte arredrada de cada torre”. (NICOLAS, 1519, fols. 91r-91v).

Pacioli resuelve el problema mediante el uso de álgebra simbólica, de modo muy diferente a la solución dada por Ventallol. Además, siguiendo a L'Huillier (1979, p. 27) ${ }^{7}$, tenemos que notar que los datos del problema de Ventallol son divisores de los datos de los problemas de Calandri y Nicolas (que son idénticos entre sí).

\subsection{La Regla 50 (fol. 131v)}

En esta regla se presenta un problema de enunciado ligeramente confuso:

"Un mestra fa huna torra enque ha 4 cambres vna sobre altra. La primera cambra es alta 20 pams. La segona es alta 15 pams. La terca es alta 10 pams. La quarta es alta 8 pams y en vn palm quadrat entren 8 pedres ious deman quantes pedres entratan en dita torra".

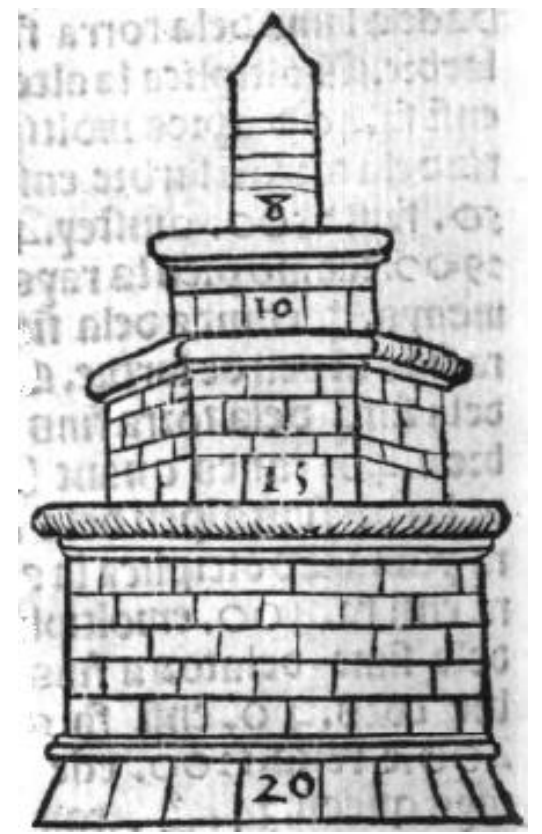

Figura 11. Ilustración correspondiente a la Regla 50.

${ }^{7}$ «D’ordinaire les médiévaux ne changent pas les valeurs numériques qu'ils trouvent dans leurs sources (...). Traditionnellement le cercle a 7 de diamètre, parfois 14; le triangle isocèle $10,10,12$ de côtes ou un multiple; le triangle équilatéral 10 de côte ou un multiple; le triangle rectangle $3,4,5$, ou un multiple, etc". 
Cuando se contempla la resolución dada por Ventallol ${ }^{8}$ se entiende que la altura de cada cámara de la torre debe coincidir con su perímetro para que el área lateral de cada una de ellas tenga el valor indicado por el autor:

En la obra de Calandri hemos encontrado dos problemas en un contexto vagamente similar al anterior:

"Uno unole amattonare una sala che e lunga 20 braccia et e larga 16 braccia et uole torre mattoni lu(n)ghi $1 / 2$ braccio et larghi $1 / 4$ dibraccio. Uo saper qua(n)ti uenenterra dentro". (CALANDRI, 1491, fol. 89r).

"Uno unol fare un muro che sia lu(n)go 20 braccia $\tau$ grosso 2 braccia $\tau$ $1 / 2 \tau$ alto 5 braccia $\tau$ 1/5 uo sapere qua(n)ti mattoni uenterra togliendo mattoni lunghi $1 / 2$ braccio $\tau$ larghi $1 / 4$ dibraccio $\tau$ grossi $1 / 8$ dibraccio". (CALANDRI, 1491, fol. 90v).

No obstante, ninguno se ajusta exactamente al que propone Ventallol en la regla 50, puesto que en ellos se debe determinar el número de ladrillos necesarios para embaldosar un suelo y para construir un muro.

\subsection{La Regla 51 (fol. 131v)}

El problema presentado en esta regla es un caso particular del siguiente problema general: Un muro de anchura $a$ rodea una superficie plana. Sabiendo que la longitud del perímetro exterior del muro es $P$, calcular la longitud del perímetro interior

"Es vna torra quadre laqual ade vogi entorn dela part de fora 50 canes ela paret es gorssa 2 canes ious deman quant haura devogi de part de dintre".

${ }^{8}\left(20^{2}+15^{2}+10^{2}+8^{2}\right) \times 8=6312$

RBHM, Vol. 16, nº 31, p. 65-90, 2016 


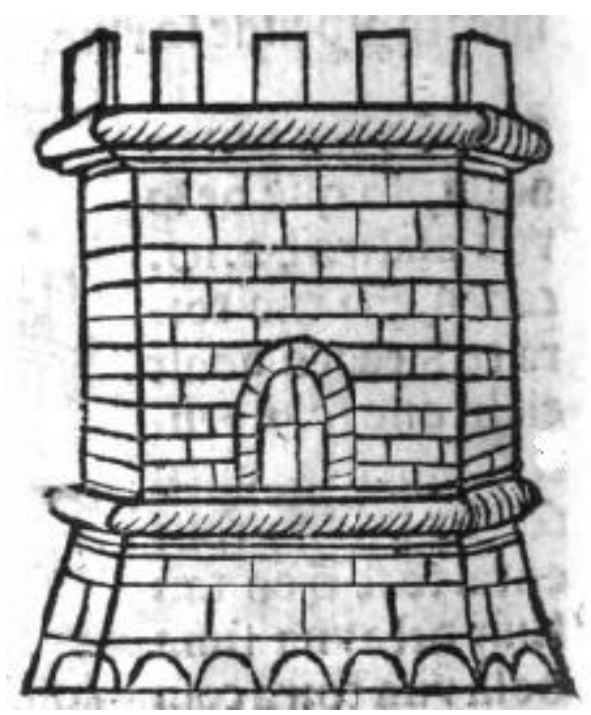

Figura 12. Ilustración correspondiente a la Regla 51.

En la Summa de Pacioli hemos descubierto un problema análogo:

"Eglie vno triangolo che 12 bracia per faccia voglio farui a ciascuna faccia vno muro grosso due bracia. adimando quanto fia il vano di dentro. Cioe quanto girera dentro per faccia". (PACIOLI, Tractatus Geometrie, fol. 56r).

El enunciado de Pacioli se corresponde con el mismo problema general, salvo que el muro tiene forma triangular frente al muro cuadrado de Ventallol.

\subsection{La Regla 52 (fol. 132r)}

El problema es idéntico al de la Regla 51, excepto por el hecho de que el muro es circular en vez de cuadrado. Valen las mismas consideraciones que en la regla anterior:

"Es vna altra torra radona la qual vogi a la part defora 44 canes e la paret de dita torra te de gruxa $3 \frac{1}{2}$ ious dema(n) qua(n)t vogi a la part de dintra".

\subsection{La Regla 53 (fol. 132r)}

Este problema involucra triángulos isósceles semejantes: 
"Son dues bigas lasquals son aiustades ensemps eson largues cascuna de 50 palms. Eyo prenc vna barra larga 6 palms la qual met enmig per axe(m)plar la vna de laltre ele tant pitiada q(ue) le feta intrar dins 20 palms ious deman quant se aperteran los caps de les bigues la hu de laltre".

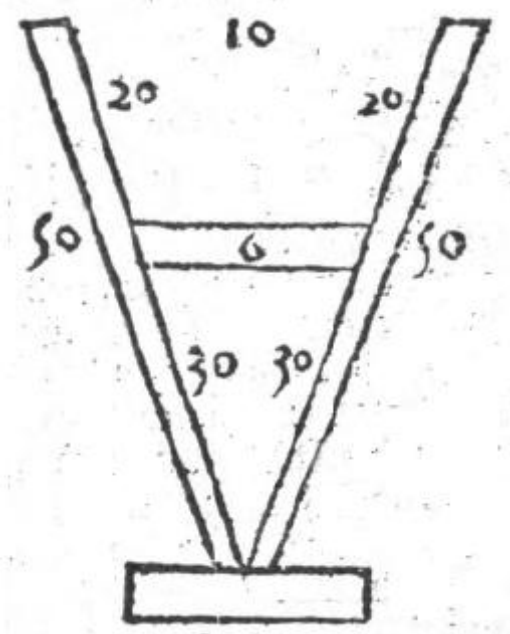

Figura 13. Ilustración correspondiente a la Regla 53.

Ninguno de los manuales consultados presenta problemas similares.

\subsection{La Regla 54 (fols. 132r-132v)}

Nuevamente nos encotramos ante un problema similar a los de las reglas 51 y 52. En este caso la figura vuelve a ser circular, aunque el contexto es diferente:

"Un mestra lauora vn arbre que gira en torn 22 palms e dit arbre te 1 palm de crosta ious dema(n) com aquesta crosta ne sia fora qua(n)t girar a lo dit arbre".

\subsection{La Regla 55 (fol. 132v)}

Este problema trata del modo en que decrece el diámetro de las secciones de un tronco de cono conforme se asciende en el mismo:

"Es hun arbre de nau que te 60 canes dalt e 15 palms de vogi per lo baix $e$ per lo alt te 3 palms de vogi ious deman quant diminuex de gruxa cascuna cana”.

RBHM, Vol. 16, n 31 , p. 65-90, 2016 

parecido.

En los libros que hemos consultado no hemos descubierto ningún problema 3.16. La Regla 57 (fols. 133r-133v)

La resolución de este problema implica el cálculo del área lateral de un cono, entre otros aspectos:

"Es vna tenda de la qual lo basto quista ficat en terra per sostener la tenda es alt 40 palms e lo drap dalt a baix te 50 palms de larc. Ious deman qua(n)ta terra cobrira aquesta tenda".

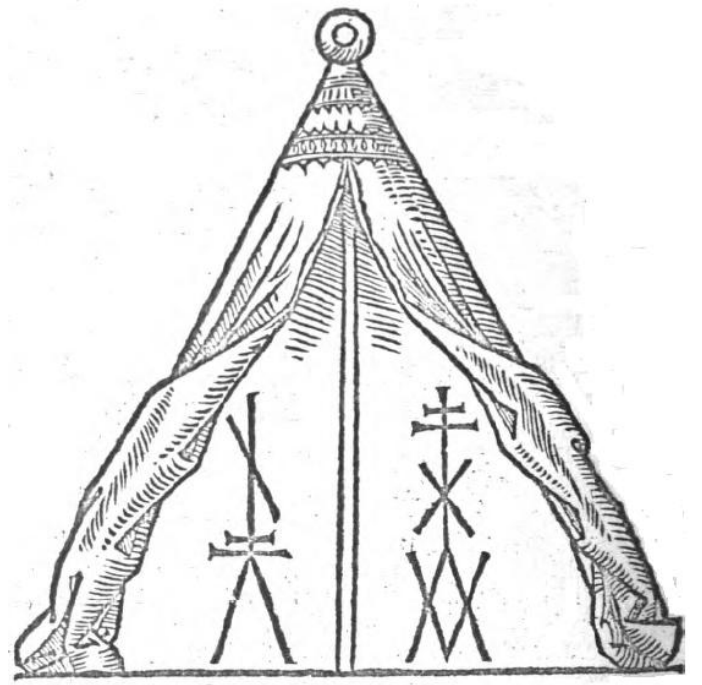

Figura 14. Ilustración correspondiente a la Regla 57.

Hemos encontrado problemas similares en Calandri, Pacioli, Juan de Ortega y Estienne de la Roche:

"Eglie u(n) padiglone che il fusto che lo reggie e alto 8 braccia e il pa(n)no qua(n)do e reso e 10 braccia cioe misurando da la pu(n)ta de lo stile pisino $i(n)$ terra.Uo sapere qua(n)te braccia di pa(n)no quadro ue dentro". (CALANDRI, 1491, fol. 96r).

"Le un padiglione che alto 8 bracia e il diametro e 12 bra(cia). Adimando qua(n)to pa(n)no ue dentro che e largo il panno 1 bra(cia) $\frac{1}{4}$ ”. (PACIOLI, 1494, Tractatus Geometrie, fol. 53r). 
"El rey nostro señor manda fazer vna tienda en vn campo a vn maestro: el qual maestro fizo la tie(n)da en que puso vn mastil q(ue) tiene de largo 100 canas y el paño que viene desde la cabeca del mastil fasta la tierra tiene de largo 140 demando que quantas canas tendra de redondez toda la tierra que torna el paño y que quantas canas haura en toda la tierra: y que quantas canas tendra el diametro de la tal tierra: y q(ue) quan(n)tas canas haura en todo el paño q(ue) cubra la tal tienda”. (ORTEGA, 1512, fols. 202v-203r).

“(...) lon veult faire vng pauillon de 11 toyses de circo(n)fere(n)ce et de troys toyses $\frac{1}{3}$ de hault despuis la circo(n)ference iusque a la pointe. Lon dema(n)de qua(n)tz aulnes de toyle y fault de troys piez $\frac{1}{2}$ de long laulne et de 2 piez $\frac{3}{4}$ de large”. (de la Roche, 1520, fol. 228r).

Para calcular el área lateral del cono $(A)$, Ventallol utiliza la fórmula incorrecta:

$$
A=\frac{L}{2} \cdot h,
$$

donde $L$ es la longitud de la base y $h$ es la altura del cono. Ésta es la misma fórmula que utiliza Ortega en su obra y, además, ambos recorren el mismo itinerario (calculo del diámetro de la base, de la longitud de la base, del área de la base y del área lateral del cono).

\section{Conclusiones}

En la sección anterior hemos recorrido los 16 problemas verbales realistas propuestos y resueltos por Ventallol en la parte geométrica de su tratado. En un buen número de ellos hemos encontrado problemas similares en los textos con los que pretendíamos compararlo.

Este análisis nos permite aventurar las posibles fuentes utilizadas por Ventallol para redactar cada uno de dichos problemas y que recogemos en la tabla siguiente.

\begin{tabular}{cc}
\hline REGLA & FUENTE \\
\hline 41 & Juan de Ortega \\
42 & Filippo Calandri \\
43 & Desconocida \\
44 & Luca Pacioli (Estienne de la Roche) \\
45 & Juan de Ortega / Gaspar Nicolas \\
46 & Filippo Calandri \\
47 & Filippo Calandri \\
48 & Luca Pacioli / Juan de Ortega / Estienne de la Roche
\end{tabular}

RBHM, Vol. 16, nº 31, p. 65-90, 2016 


\begin{tabular}{lc}
49 & Filippo Calandri / Gaspar Nicolas \\
50 & Filippo Calandri \\
51 & (Luca Pacioli) \\
52 & (Luca Pacioli) \\
53 & Desconocida \\
54 & (Luca Pacioli) \\
55 & Desconocida \\
57 & Juan de Ortega \\
\hline
\end{tabular}

En la mayor parte de los casos analizados, no sólo los problemas son similares, sino que los problemas se presentan en un contexto idéntico. Tal es el caso, por ejemplo, de la Regla 44 en la que el contexto involucra una torre, un río y una escalera como elementos fundamentales.

En muchos casos se aprecia también una clara relación entre los datos numéricos de los problemas. En la ya citada Regla 44, por ejemplo, Ventallol maneja un triángulo rectángulo de lados 6, 8 y 10 mientras que Pacioli maneja un triángulo rectángulo de lados 30, 40 y 50. Ambos son múltiplos del triángulo rectángulo "elemental” de lados 3, 4 y 5 . Del mismo modo, en la Regla 49, los datos del problema de Ventallol son divisores de los datos del problema (idéntico) presentado por Calandri y Nicolas.

En ocasiones hay que buscar las similitudes (y por tanto las diferencias) en los métodos de resolución. Por ejemplo, en las Reglas 46 y 57 existen varios autores que proponen problemas similares y es el método de resolución utilizado lo que nos permite inclinarnos por uno de ellos.

En otras situaciones (Reglas 51, 52 y 54) el contexto es idéntico pero Ventallol plantea los problemas utilizando un cuadrado o un círculo en lugar de un triángulo. En un caso (Regla 50) la única similitud radica en un contexto vagamente similar. Finalmente, desconocemos si las reglas 43, 53 y 55, son originales del autor mallorquín o provienen de otras fuentes.

En definitiva, a la vista de estos datos, parece que los problemas geométricos realistas de enunciado verbal contenidos en el sexto tratado de la "aritmética" de Ventallol, se inspiran principalmente en las obras de Calandri, Pacioli y Ortega. La influencia de los textos de Nicolas y de la Roche no es concluyente y, en el mejor de los casos es de un grado mucho menor.

\section{Referencias bibliográficas}

BEAUJOUAN, G. (1988). The place of Nicholas Chuquet in a tipology of fifteenth-century French arithmetics. En C. Hay (Ed.), Mathemtics from manuscript to print (1300-1600), (pp. 73-88). Oxford: Clarendon Press.

BOVER DE ROSSELLÓ, Joaquín María. (1838). Memoria biográfica de los mallorquines que se han distinguido en la antigua y moderna literatura. Palma: Imprenta Nacional regentada por D. Juan Guasp. 
CALANDRI, Filippo (1491). De arimethrica opusculum. Firenze: Lorenzo de Morgiani e Giovanni [di Piero] da Maganza.

CÉU SILVA, M. (2013). Renaissance sources of Juan Pérez de Moya's Geometries. Asclepio. Revista de Historia de la Medicina y de la Ciencia, 65(2), p018.

DIAZ, M.V. y POBLETE, A. (2001). Contextualizando tipos de problemas matemáticos en el aula. Números, 45, pp. 33-41.

DOCAMPO REY, J. (2006). Reading Luca Pacioli’s Summa in Catalonia: An early 16thcentury Catalan manuscript on algebra and arithmetic. Historia Mathematica, 33, pp. 4362.

LABARTHE, M.H. (2004). Premières arithmétiques imprimées des Espagnes: Une hiérarchie des problèmes au service des procédés de résolution. Tesis doctoral. Université Paul Sabatier, Toulouse III.

LAMASSÉ, S. (2015). Mise en série et tradition de problèmes dans les arithmétiques en langue vulgaire de la fin du Moyen Âge. En A. Bernard (Ed.). Les séries de problèmes, un genre au carrefour des cultures. París : HASTEC.

L'HUILLIER, Hervé. (1979). Nicolas Chuquet. La Géométrie. París: Vrin.

MASSA, M.R. (2012) "Spanish Arte Mayor in the Sixteenth Century". En S. Rommevaux, M. Spiesser y M.R.Massa (dir.). Pluralité de l'algèbre à la Renaissance entitled, pp. 103126. París: Honoré Champion.

NICOLAS, Gaspar (1541). Tratado da pratica Darismetica (Terecera edición). Luis Rodriguez.

ORTEGA, Juan de (1512). Conpusicion de la arte de la arismetica y juntamente de geometría. León: en casa de Maistro Nicolau de Benedictis: por Joannnes Trinxer librero de Barcelona.

PACIOLI, Luca (1494). Summa de Arithmetica, Geometria, Proportioni et Proportionalita. Veneza: Paganini.

PIFERRER, Francisco. (1858). Nobiliario de los reinos y señoríos de España (Tomo IV). Madrid: Imprenta de M. Minuesa.

REY PASTOR, Julio (1926). Los matemáticos españoles del siglo XVI. Biblioteca Scientia. ROCHE, Estienne de la (1520). Larismethique nouellement composee. Lyon: Constantin Fradin.

TOLRA, Juan Bautista (1619). Arismetica de Ivan Ventallol, tradvzida de lengva Catalana en Castellana, por el Doctor Juan Batista Tolra. Va añadido vn Tratado de la ARTE MAYOR llamada ALGEBRA o RREGLA DE LA COSA compuesto por el mismo Doctor Tolra. Tarragona: Gabriel Roberto.

USÓN, C. (2004). Aritméticas aragonesas del s. SVI. A la sombra del Quadrivium. Actas del VIII Congreso de la Sociedad Española de Historia de las Ciencias y de las Técnicas, pp. 453-471.

VENTALLOL, Joan, (1521). Pratica mercantíuol. Lyo: Joan de la Place.

VENTAYOL SUAU, Pedro. (1974). "El apellido Ventayol, en Alcudia". Bolletí de la Societat Arqueològica Lul-liana. Vol. 34, pp. 319-326. 
Vicente Meavilla Seguí

Catedrático de Matemáticas jubilado

Doctor en Filosofía (Pedagogía)

E-mail: vmeavill@hotmail.com

Antonio M. Oller Marcén

Centro Universitario de la Defensa

Academia General Militar

E-mail: oller@unizar.es 